\title{
Phason Relaxation Mechanisms in Al-Pd-Mn Single-Quasicrystals
}

\author{
M. Weller, M. Feuerbacher*, J. Diehl and K. Urban* \\ Max-Planck-Institut für Metallforschung, Institut für Werkstoffwissenschaft, Seestrasse 92, \\ 70174 Stuttgart, Germany \\ * Institut für Festkörperforschung, Forschungszentrum Jülich GmbH, 52425 Jülich, Germany
}

\begin{abstract}
Quasicrystals represent a new state of condensed matter which exhibits long-range quasiperiodic translational order and orientational order with noncrystallographic symmetry. Mechanical spectroscopy was applied for the study of defect properties in stable icosahedral single-quasicrystalline AlPd-Mn samples. Two mechanical loss maxima appear at $360 \mathrm{~K}$ and $870 \mathrm{~K}$ for a measuring frequency of $\mathrm{f} \approx 3 \mathrm{~Hz}$. The relaxation parameters for both peaks were determined as $\mathrm{H}=0.98 \mathrm{eV}, \tau_{\infty}{ }^{-1}=4.5 .10^{14} \mathrm{~s}^{-1}$ for the low temperature peak, and $\mathrm{H}=4.0 \mathrm{eV}, \tau_{\infty}^{-1}=3.0 \cdot 10^{24} \mathrm{~s}^{-1}$ for the high temperature peak, respectively. Both peaks are related to phason defects which are characteristic for the quasicrystalline structure.
\end{abstract}

\section{INTRODUCTION}

Quasicrystals, a shortened term for quasiperiodic crystals, represent a new state of matter with respect to order and symmetry. Shechtman et al. [1] observed in quenched AlMn alloys diffraction patterns with rotational axes of 5-fold, 3-fold and 2-fold symmetry which are forbidden for translational symmetry in crystalline solids. Quasicrystals exhibit long-range quasiperiodic translational order and long-range orientational order with disallowed symmetry. Since the discovery of the icosahedral AlMn phase a large number of quasicrystalline alloys has been discovered (for reviews see eg. [2-4]).

As for crystalline solids quasicrystals may contain imperfections, i.e. defects, of different type such as point defects (vacancies, interstitial atoms) or dislocations. Additionally, a new type of "point defect" must be introduced, the phason defect. An ideal quasiperiodic structure may be represented by an arrangement of tiles according to strict matching rules in an abstract 3-dimensional Penrose lattice. Phasons represent imperfections by local rearrangement of tiles, corresponding to discrete displacements of atoms.

Not much is known on the anelastic properties of quasicrystals. A few internal friction experiments are reported on poly-quasicrystalline Al-based quasicrystals. Van Cleve et al. [5] observed a large linear increase of internal friction with temperature in the $1-100 \mathrm{~K}$ range. Okumura et al. [6] found a mechanical relaxation peak in poly-quasicrystalline icosahedral $\mathrm{Al}-\mathrm{Cu}-\mathrm{V}$ at $440 \mathrm{~K}(10 \mathrm{~Hz})$ which they attributed to phasons. A weak point of experiments on poly-quasicrystalline samples, which were prepared by quenching, is that grain boundaries, and possibly second phases, may contribute to the mechanical loss spectra. With the discovery of thermodynamically stable quasicrystalline phases [7] which allow for the Czochalski growth of large single-quasicrystals it is now possible to obtain centimetre-sized single phase specimens for mechanical loss spectroscopy. First experiments on Al-Pd-Mn single-quasicrystals show mechanical loss peaks which are correlated to the quasicrystalline structure, i.e. phason defects [8]. 


\section{EXPERIMENTAL}

A single-quasicrystal of about $10 \mathrm{~cm}$ in length and $0.8 \mathrm{~cm}$ diameter with the composition 70.3 at. $\% \mathrm{Al}$ 21.5 at.\% $\mathrm{Pd}-8.2$ at.\% $\mathrm{Mn}$ was grown by the Czochalski technique. The single-quasicrystallinity and structural perfection was checked by various methods such as Laue diffraction, electron diffraction in transmission electron microscopy, and scanning electron microscopy [8]. The dislocation density was determined to about $10^{7} \mathrm{~cm}^{-2}$ [9]. Samples with dimensions of $50 \times 5 \times 1 \mathrm{~mm}^{3}$ were cut from the quasicrystal by spark erosion, with edges parallel to twofold axes. According to infrared spectroscopy the samples contained about 17 at.ppm $\mathrm{N}$ and less than 130 at.ppm C. The oxygen content varied between 15 at.ppm and 1200 at.ppm in different samples. The internal friction measurements were carried out with two types of apparatus operating in different frequency ranges using the same sample. (i) Low frequency (2-12 $\mathrm{Hz}$ ) measurements were carried out with an inverted torsion pendulum [10]. For thermal exchange He-gas with a pressure of $\approx 10$ mbar was used. (ii) High frequency measurements in the $\mathrm{kHz}$ range were performed by exciting the horizontally held specimens in flexural eigenvibrations [11]. The mechanical part operating under high vacuum conditions $\left(10^{-4}\right.$ mbar) is restricted to measurements below $800 \mathrm{~K}$.

\section{RESULTS}

Figure 1 shows the internal friction, $Q^{-1}$, and the frequency square, $f^{2}$, versus temperature $T$ in the range of $200 \mathrm{~K}$ to $1070 \mathrm{~K}(\mathrm{f} \approx 3 \mathrm{~Hz}$ ). The dashed curves were obtained in a second run. The loss spectra exhibit two distinct peaks designated with $\mathrm{A}$ and $\mathrm{B}$. The high temperature peak at $\mathrm{T}_{\mathrm{m}} \approx 870 \mathrm{~K}$ is about two orders of magnitude larger than the low temperature peak at $\mathrm{T}_{\mathrm{m}} \approx 360 \mathrm{~K}$. In the second run the peaks occur at the same positions with approximately the same height as in the first run. A small additional shoulder occurs at $\approx 960 \mathrm{~K}$ and the shear modulus $\left(\mathrm{f}^{2}\right)$ values below $680 \mathrm{~K}$ are increased by about $3 \%$.

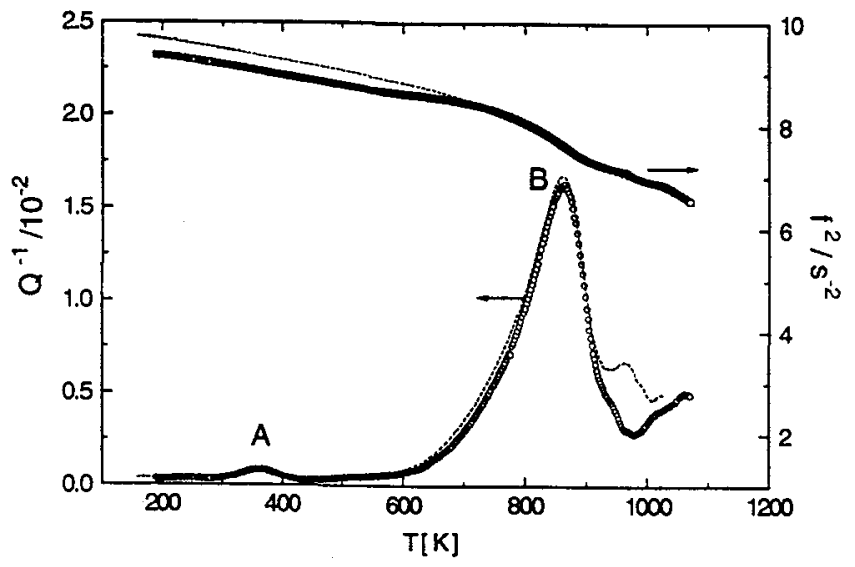

Figure 1: $\mathrm{Q}^{-1}$ and $\mathrm{f}^{2}$ vs. temperature for Al-Pd-Mn $(\mathrm{f} \approx 3 \mathrm{~Hz})$. Dashed: second run after heating to $1070 \mathrm{~K}$.

Fig. 2 shows the $\mathrm{Q}^{-1}(\mathrm{~T})$ data of peak A from Fig. 1 in comparison with a measurement at $3.3 \mathrm{kHz}$ of the same specimen. The peak position is shifted to higher temperature $\left(T_{m}=465 \mathrm{~K}\right)$ with increasing frequency. The position of the high-temperature peak $B$ is also frequency dependent. These results indicate that both peaks are associated with thermally activated relaxation processes. From the temperature shift the relaxation parameters listed in Table 1 were determined. Both peaks are broader than theoretical Debye peaks calculated with the relaxation parameters from Table 1 . Values of the half width broadening are included in Table 1. Peak B is asymmetrically broadened on its low temperature side. 


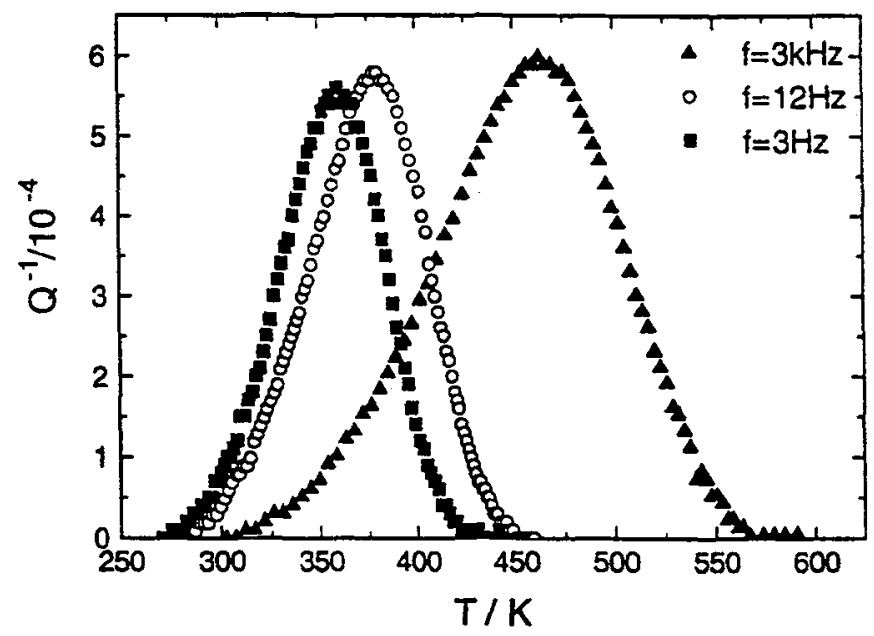

Figure 2: Temperature dependence of peak A for various different frequencies after subtraction of background damping $(3 \mathrm{~Hz}, 12 \mathrm{~Hz}$, and $3.1 \mathrm{kHz}$ ).

\begin{tabular}{|c|c|c|c|}
\hline & IV(e) & (1) & (1) \\
\hline Peak A & $0.98 \pm 0.02$ & $4.5 \cdot 10^{14 \pm 1}$ & 2 \\
\hline Peak B & $4.0 \pm 0.4$ & $3.0 \cdot 10^{24 \pm 1}$ & 3 \\
\hline
\end{tabular}

Table 1: Relaxation parameters of peaks $A$ and $B$

\section{DISCUSSION}

The mechanical loss spectra of icosahedral Al-Pd-Mn single-quasicrystals exhibit two distinct loss maxima, $\mathrm{A}$ and $\mathrm{B}$. Both maxima are stable to annealing and originate from thermally activated relaxation processes.

Peak $A$ is controlled by an attempt frequency, $\tau_{\infty}{ }^{-1}$, in the range of $10^{14} \mathrm{~s}^{-1}$ which is characteristic for reorientation of point defects by single-atomic jumps. At first sight, one may think that peak $A$ could be due to a Snoek-type relaxation similar to that of interstitial solute atoms $(\mathrm{O}, \mathrm{N}, \mathrm{C})$ in bcc metals. Since, however, the peak height is independent of the foreign atom content at all concentrations for which measurements were performed, we reject this interpretation in favour of an intrinsic relaxation mechanism.

In quasicrystals stress-induced phason jumps could provide a relaxation mechanism whereby atomic jumps over small distances are involved. Phasons may be visualized as local rearrangement of tiles within the abstract three-dimensional (3D) Penrose lattice. These defects occur naturally in quasicrystals but they can also be introduced by plastic deformation [9]. Phason defects are illustrated in Fig. 3 in a 2D-model. The Penrose lattice appears as a plane filling of two types of rhombi with equal edges, one with angles of $36^{\circ}$ and $144^{\circ}$ (skiny rhombus, S), and the other with angles of $72^{\circ}$ and $108^{\circ}$ (fat rhombus, F). The tiling has to obey distinct matching rules. Such an array is shown in Fig. 3. The shaded part of this array contains phason defects. Fig. 3a shows this arrangement with two hexagons (left and right) for the ideal Penrose lattice. The right side depicts a phason defect which is formed by rearrangement of the fat and skiny rhombi in the right hexagon. Such a misfit configuration is formed by "flipping" of the segments within the hexagous from position (1) to (2) and represents a phason. This flip, in terms of the atomic decoration, corresponds to a single atomic jump. The "atom" has to cross an energy barrier between the two positions $(1,2)$. Thus the relaxation process is controlled by the corresponding phason diffusion process [13]. It can be performed by short range motion of atoms and requires no vacancies to be present. Similar flipping configurations can be imagined within fat rhombi configuration $(3,4)$ in $(b)$. We propose that peak $A$ is due to stress induced phason jumps. This relaxation process is based on single atomic jumps which are characteristic for point defects. Phason jumps have been postulated to be the basis of a mechanism for self diffusion in quasicrystals, and the activation energy is stated to be low [13]. 


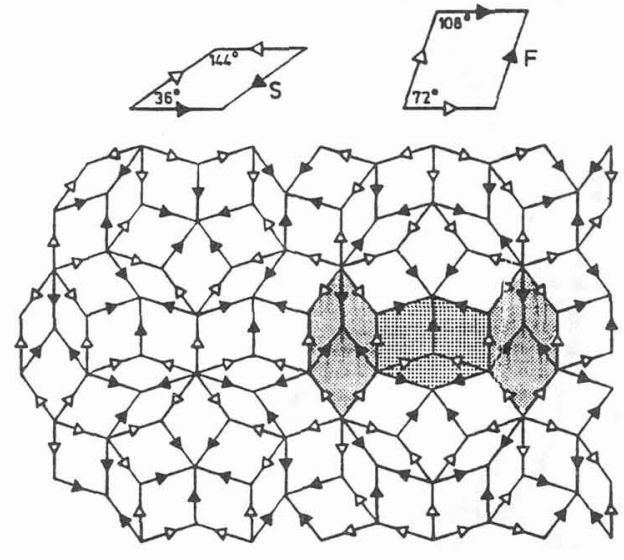

(a)
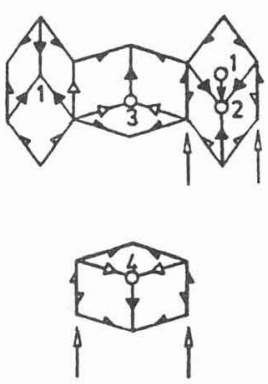

(b)

Figure 3: (a) 2D-Penrose lattice with Phason defects (shaded area). (b) Phason flip within a hexagonal cluster. Violation of the marching rules is indicated with arrows.

The attempt frequency $\tau_{\infty}{ }^{-1}$, of peak $B$ is about 10 orders of magnitude larger than that of peak $A$. This indicates that the underlying relaxation mechanisms involves collective motion of atomic defects (similar as e.g. for the Snoek-Köster relaxation in bcc metals). Collective motion of atoms was recently found to be the controlling mechanism for self-diffusion in amorphous metallic alloys [14], where a pre-exponential factor of up to 12 orders of magnitude larger than in crystalline alloys was found. By analogy we propose that the collective motion of a larger-number of atoms is also involved in the relaxation mechanism of peak B. In terms of phason defects this means that whole chains or larger worms of phason type rearrangements are involved. Such collective phason excitations were discussed theoretically by Trub and Trebin [15] in connection with self-diffusion in quasicrystals.

Further experiments are in progress to arrive at a more conclusive picture of the atomistic relaxation processes which determine mechanical loss spectra of quasicrystals.

\section{References}

[1] Shechtman, D., Blech, I., Gratias, D., and Chan, J.W, Phys.Rev.Lett. 53 (1984) 1951-1953.

[2] Kelten, K.F., Quasicrystals: Structure and Stability. Intern. Mater. Reviews 38 (1993) 105-137.

[3] Lectures on Quasicrystals, Hippert, F. and Gratias, D., Eds. (Les Editions de Physique, Les Ulis, 1994).

[4] Janot, J., Quasicrystals: a primer, 2nd ed., (Clarendon Press, Oxford, 1994).

[5] Van Cleve, J.E., Gershenfeld, N.A. Knorr, K., and Bancel, P.A., Phys.Rev. B41 (1990) 980-990.

[6] Okumura, H., Tsai, A.P., Inoue, A., and Masumoto, T., Mater.Sci. Eng. A181/182 (1994), 781-784.

[7] Tsai, A.P., Inoue, A., and Masumoto, T., Mater. Trans. Japan Inst. Metals 30, (1989) 666-676.

[8] Feuerbacher, M., Weller, M., Diehl, J., and Urban, K., Phil.Mag.Lett., in press.

[9] Rosenfeld, R., Feuerbacher, M., Baufeld, B., Bartsch, M., Wollgarten, M., Hanke, G., Beyss, M., Messerschmidt, U., and Urban, K., Phil. Mag. Leit. 72 (1995) 375-384.

[10] Weller, M., J. de Physique, IV, 5, C7 (1995) 199-204.

[11] Weller, M., and Török, E., J. de Physique 48, C8 (1987) 371-376.

[12] Scolar, E.S.J., Lubensky, T.C., and Steinhard, P.J., Phys.Rev. B 34, (1986) 3345-3360.

[13] Kalugin, P.A., and Katz, A., Europhys. Lett. 21.9 (1993) 921-926.

[14] Frank, W., Hörner, A., Scharwaechter, P., and Kronmüller, H., Mat.Sci.Eng.A179-180 (1994) 36-41.

[15] Trub, A., and Trebin, H.R., J. de Physique I, 4 (1994) 1855-1866. 